\title{
A PARALLEL IMPLEMENTATION FRAMEWORK FOR REMOTELY SENSED IMAGE FUSION
}

\author{
Yang Jinghui*, Zhang Jixian \\ Chinese Academy of Surveying and Mapping, Lianhuachi West Road 28, Beijing 100830, P. R. China \\ *: Corresponding author. Email: jhyang@casm.ac.cn, jhyang@vip.163.com.
}

\section{Commission VII, WG VII/6}

KEYWORDS: Remote Sensing, Image Fusion, Parallel Computing

\begin{abstract}
:
The fusion algorithms are data-intensive and computation-intensive, whose processing performance is low if common implementation techniques are adopted. This paper presents a parallel implementation framework based on a generalized fusion model and parallel computing for remotely sensed image fusion. The steps in the implementation corresponding to each fusion algorithm are mainly the same, which discard the process steps, input and output ( I/O) operations not impacting the last results. The parallel processing mechanism adopts the data decomposition strategies where different data blocks can be processed in different CPU cores. The developed software module shows that the framework can be applied to implement many algorithms which usually are divided into three general categories. The experiments show that the parallel implementation framework is high performance, which not only decreases the processing time in single CPU core environment but also efficiently utilizes the resources of multi-cores in computer.
\end{abstract}

\section{INTRODUCTION}

Image fusion is capable of integrating different imagery to produce more information than can be derived from a single sensor, in which the lower resolution multispectral image's structural and textural details are enhanced by adopting the higher resolution panchromatic image corresponding to the multispectral image. So far, many pixel level fusion methods, for remote sensing images have been presented.

However some algorithms are very complicated, it is very difficult to implement them for us. The reasons are that these algorithms need many steps and generate temporary files in order to implement them, although some processing steps and temporary files do not impact the last results. Thus, a very undesirable result is that the execution of fusion methods which usually using serial processing is very slowly. The processing efficiency sometimes is the determinant aspect for their broad applications.

In order to resolve these problems, we present a parallel implementation framework for remotely sensed image fusion, which mainly rely on two improvements. One is the implementation techniques based on the generalized model (Yang, et al., 2009, Zhang and Yang, 2008) for image fusion. The framework can discard the middle steps and I/O operations not impacting the last results for some fusion algorithms because of directly calculating the last fusion results. The other is the framework is coupled with parallel computing. The adopted parallel processing technique can fully utilize the computing resource of the processing platforms, which are mostly multi CPU cores machine or cluster computers. The parallel processing mechanism can divide an entire image into different blocks which are dispatched to different processing units. Thus the processing efficiency is extremely improved. The framework can be applied to most image fusion algorithms, which are divided into three general categories (Yang, et al., 2010), i.e., component substitution (CS) fusion technique, modulation-based fusion technique and multi-resolution analysis (MRA) based fusion technique.

\section{THE PROPOSED PARALLEL IMPLEMENTATION FRAMEWORK}

The framework mainly consists of two important features. One is based on the generalized fusion model (Yang, et al., 2009, Zhang and Yang, 2008), the other is the parallel proceeding mechanism. Meanwhile, the two features are coupled completely.

\subsection{The proposed framework}

According to the generalized fusion model (Yang, et al., 2009, Zhang and Yang, 2008), the fusion results for most fusion algorithms is formulated as:

$$
x s_{(k, i, j)}^{H}=x s_{(k, i, j)}^{L}+\alpha_{(k, i, j)} \cdot \delta_{(i, j)}
$$

$x S_{(k, i, j)}^{H}$ is intensity value of the $(i, j)$ pixel of the fused kth band; $X S_{(k, i, j)}^{L}$ is intensity value of the $(i, j)$ pixel of the lower resolution multispectral $k$ th band; $\delta_{(i, j)}$ is spatial and textural details extracted from the panchromatic band by a 
certain calculation; and $\alpha_{(k, i, j)}$ is the coefficients modulating $\delta_{(i, j)}$ into $x S_{(k, i, j)}^{L}$. From the formula (1), the main steps corresponding to each fusion algorithm are almost the same when those algorithms are implemented, i.e., calculating the parameters $\delta_{(i, j)}$ and $\alpha_{(k, i, j)}$. Since three general categories of fusion algorithms (Yang, et al., 2010) can be deduced to the generalized model by mathematical transformation, the framework can be applied to them. In addition, Yang et al. (2009) lists the computing methods of the two important parameters in the model for most commonly used fusion algorithms. Therefore the basic implementation framework is established. The advantages of the implementation framework lie in two aspects. One is to avoid a specific implementation method for a specific fusion algorithm. Most fusion algorithms are implemented according to the last fusion results, which have the same parameters. The other is that the implementation framework can discard the middle steps and I/O operations not impacting the last results for some fusion algorithms because of directly calculating the last fusion results.

The most important characteristic is that parallel computing can easily be incorporated into the framework. The parallel proceeding mechanism is shown in Fig. 1. The master processor receives the results sent by multi-slave processors and then writes the results to the output file, whereas the slave processors read data blocks from the input file according to a specific data decomposition strategy and then proceed these respective data blocks. The data decomposition strategies adopted in the framework are shown in Fig.2. An entire image can be divided into strips (Fig. 2a), tiles (Fig. 2b) or bands (Fig. 2c) respectively. One among these three types of strategies can be chosen according the real requirements.

In a word, the framework only calculates the two parameters $\delta_{(i, j)}$ and $\alpha_{(k, i, j)}$ in the formula (1) instead of processing step by step according to the generalized fusion model, meanwhile it can concurrently process multiple data blocks by adopting the data decomposition strategy.

\subsection{The developed software module}

A software module has developed for image fusion based on the implementation framework. The eight algorithms respectively using Intensity Hue Saturation (IHS) (Carper, 1990, Shettigara, 1992), Color Normalization (CN) (Vrabel, 2000), Principal Component Analysis (PCA) (Shettigara, 1992), Block Regression (BR) (Zhang and Yang, 2010), Smoothing Filter Based Intensity Modulation (SFIM) (Liu, 2000), Local Correlation Modelling (LCM) (Hill, 1999), additive à trous wavelet transform (N'ũnez, 1999), and combing PCA and à trous wavelet transform (González-Audícana, et al, 2004), which all supports parallel computing. The GUI of the software module is shown in the
Fig. 3. From top to down, the function of the GUI is for selecting the processing number, selecting the panchromatic image, selecting the multispectral image, choosing a fusion algorithm, selecting output fused file, and the bottom is for displaying the processing status information. The software module can supports not only single machine with multicore CPU but also HPC (high performance computing) computers. It can run on different operation systems, e.g. Microsoft Windows, Linux and Mac OS X.

\section{EXPERIMENTS}

We take two kinds of experiments. One is to record the execution time which can demonstrate the efficiency of parallel processing mechanism of Fig.1 in comparison with serial processing. The other is to demonstrate the fusion quality in comparison with commercial software ERDAS.

\subsection{Parallel Processing Experiments}

The experiments are tested in an ordinary PC with 4 cores running on Windows 7 operation systems. The panchromatic and multispectral images in the same scene are fused respective by using IHS transform fusion algorithm in the software, SFIM fusion algorithm in the software and IHS transform fusion algorithm in ERDAS software. The file size of the fused image is 3.1 Gigabyte. The process time with different cores are indicated in Tab. 1. The time using IHS method in the framework is less than the same method in the ERADS software. The reason is that the framework discards the middle steps and I/O operations not impacting the last results. In this case of single machine, the time using IHS in the framework is near one ninth of time using ERDAS software. The speedup of SFIM and IHS in framework is near $2.5 \mathrm{x}$ when four cores are used. The more are the CPU cores used, the high speedup. The experiments show that the parallel implementation framework for remotely sensed image fusion is high performance, which not only decreases the processing time in single CPU core environment but also efficiently utilizes the property of multi-cores in computer.

\subsection{Visual Comparison of Fusion Results}

The other test is to demonstrate the visual effect of fusion result by comparing with the commercial software ERADS. Fig. 4(a) and Fig. 4(b) show QuickBird panchromatic and multispectral image respectively. Fig. 4(c) and Fig. 4(d) show the fusion results of BR and IHS fusion algorithms in the framework respectively. Fig. 4(e) shows fusion results of MOD. IHS Resolution Merge algorithm in ERDAS. All these fusion result are displayed in the form of color composite of 4 , 3, 2 bands. From the Fig.4, the BR results are relatively better than IHS fusion algorithms in the framework and ERADS. The fusion results of two different implementations of IHS algorithm are slightly different, the maintenance of spectral 
characteristics of Fig. 4(e) is better than Fig. 4(d), whereas the enhancement of spatial details of Fig. 4(d) is better than
Fig. (e)

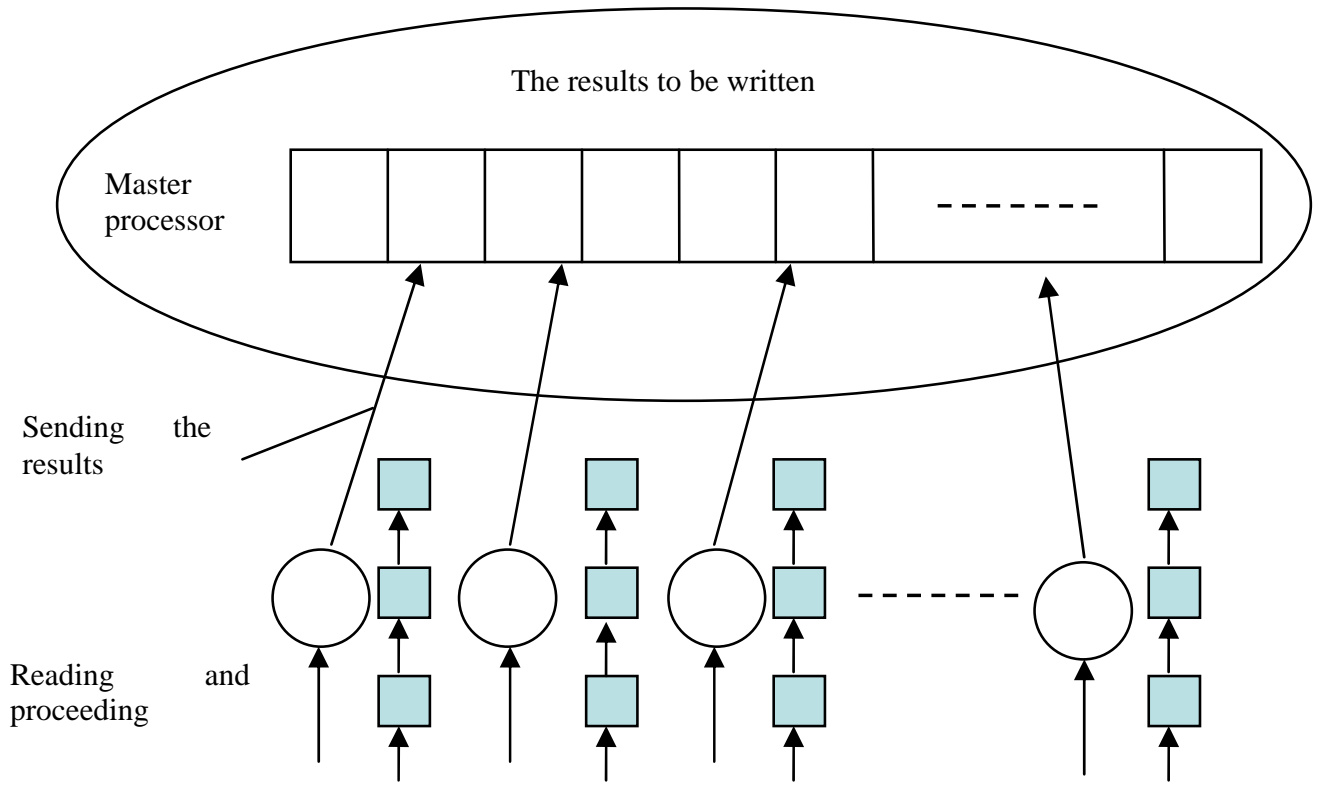

Slave processors

Fig. 1 The parallel proceeding mechanism

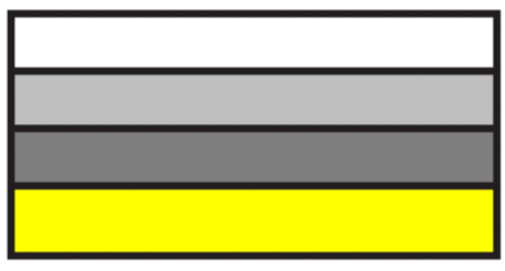

(a)

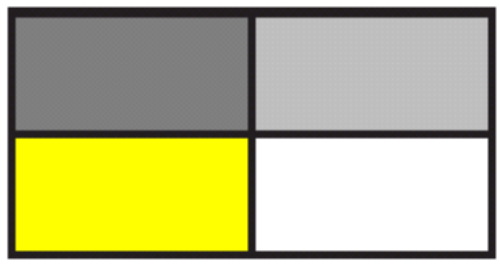

(b)

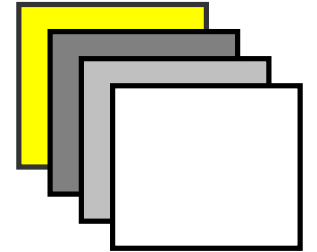

(c)

Fig. 2 Three types of data decomposition strategies adopted in the framework. (a) Strip; (b) Tile; (c) Band.

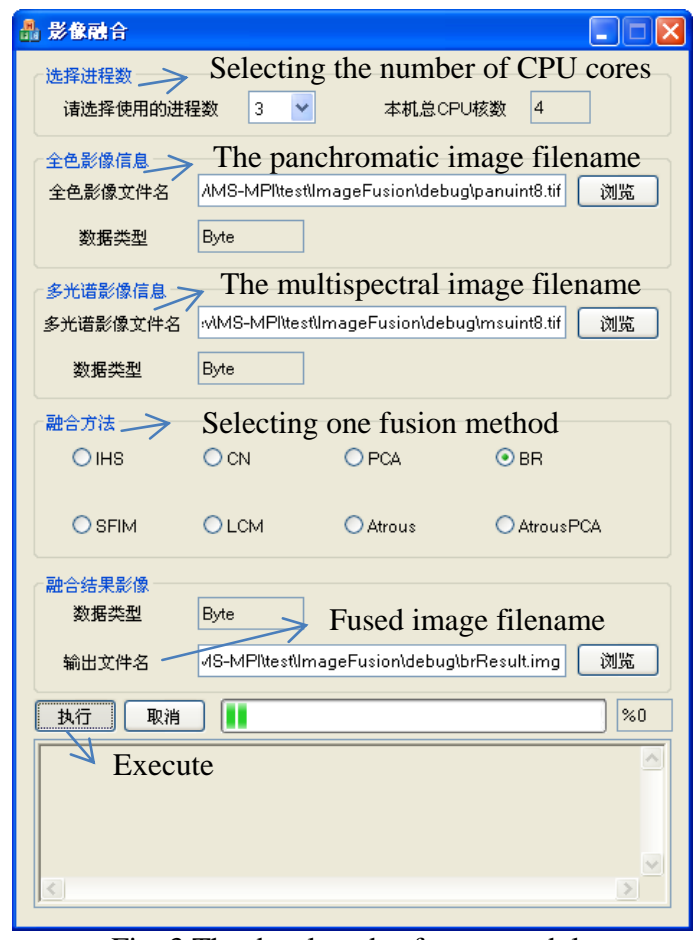

Fig. 3 The developed software module 
ISPRS Annals of the Photogrammetry, Remote Sensing and Spatial Information Sciences, Volume I-7, 2012 XXII ISPRS Congress, 25 August - 01 September 2012, Melbourne, Australia

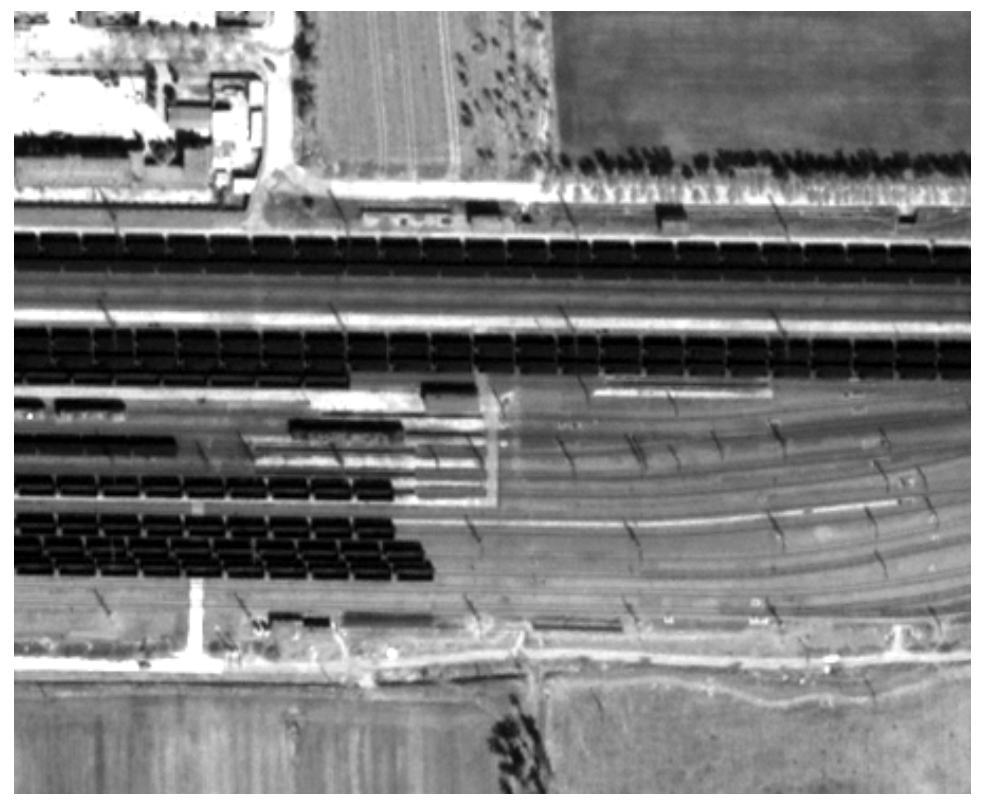

(a)

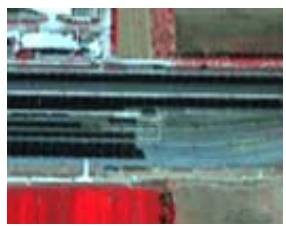

(b)

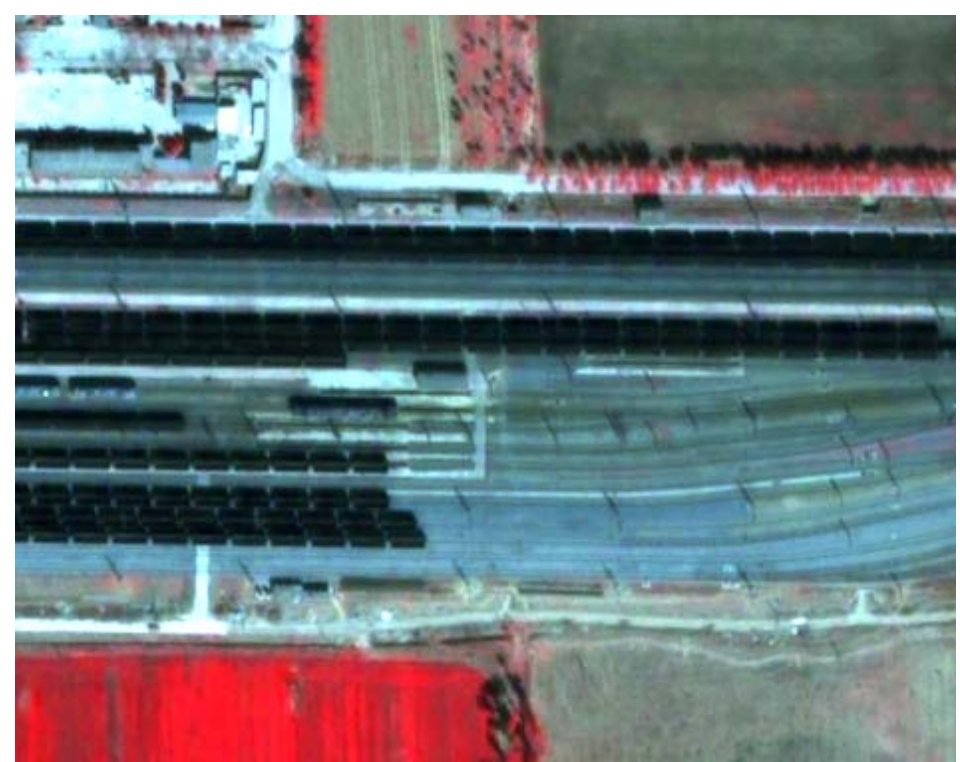

(c) 


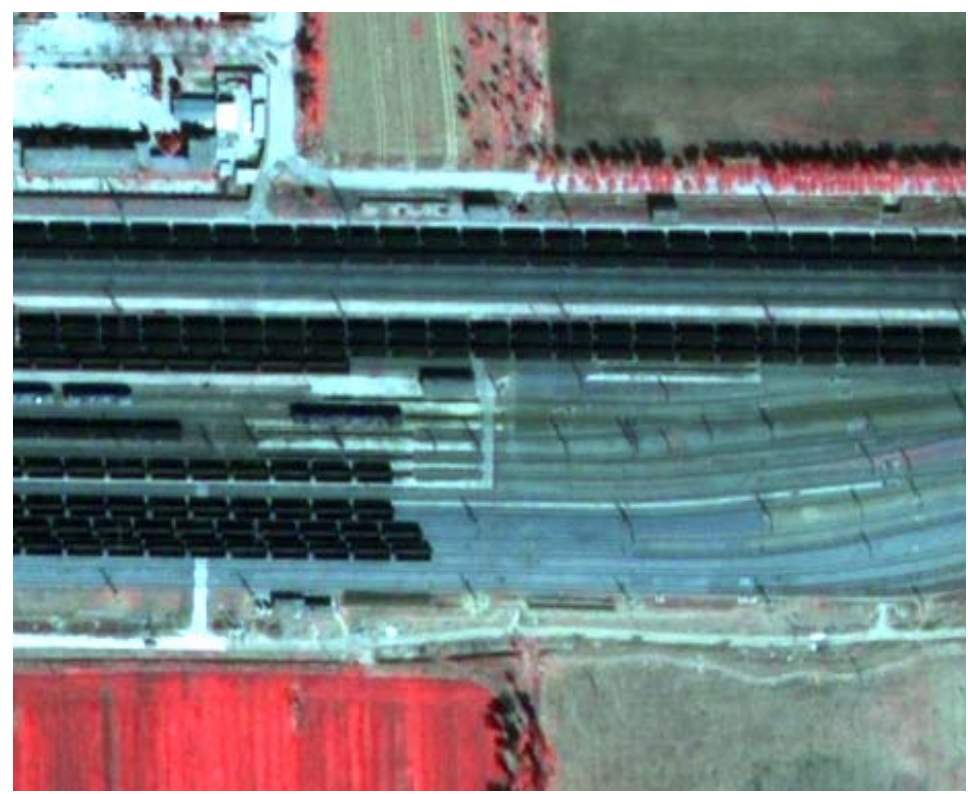

(d)

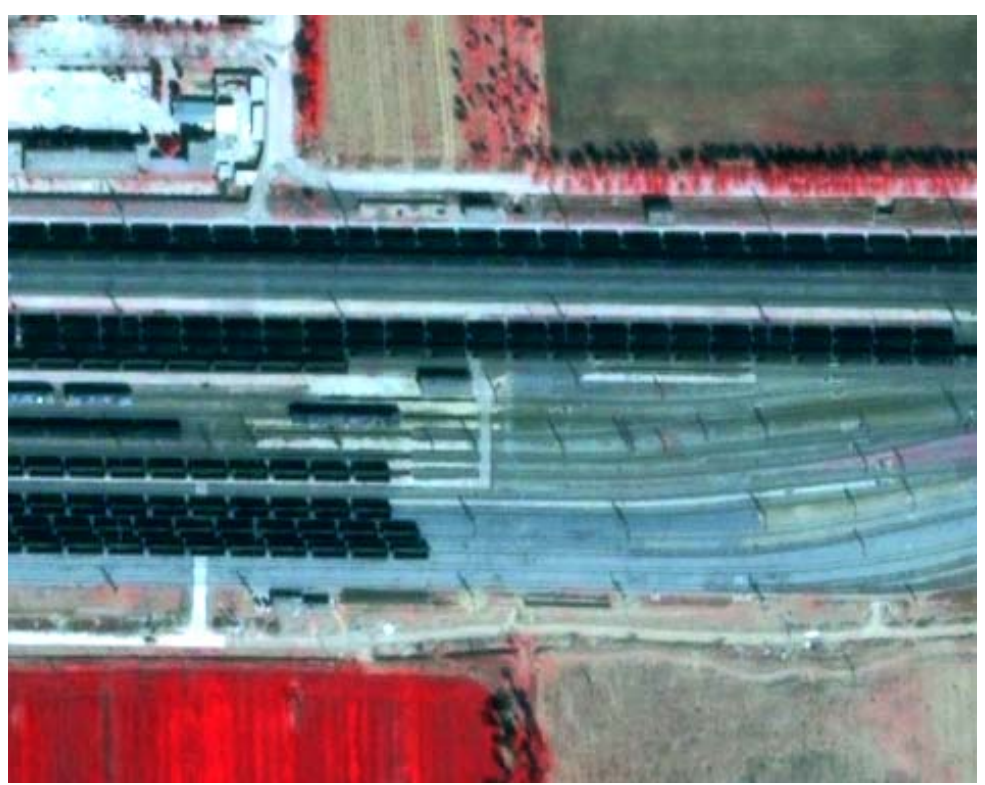

(e)

Fig. 4 Visual Comparison of Different Algorithms. (a) QuickBird panchromatic image; (b) QuickBird multispectral image; (c) Fusion result of BR algorithm in the framework, color composite of 4, 3, 2 bands; (d) Fusion result of IHS fusion algorithm in the framework, color composite of 4, 3, 2 bands; (e) Fusion result of MOD. IHS Resolution Merge algorithm in ERDAS, color composite of 4, 3, 2 bands.

\begin{tabular}{|c|c|c|c|c|}
\hline cores & 1 & 2 & 3 & 4 \\
\hline SFIM & $673 \mathrm{~s}$ & $455 \mathrm{~s}$ & $397 \mathrm{~s}$ & $358 \mathrm{~s}$ \\
\hline IHS & $250 \mathrm{~s}$ & $202 \mathrm{~s}$ & $159 \mathrm{~s}$ & $109 \mathrm{~s}$ \\
\hline IHS (ERDAS) & $897 \mathrm{~s}$ & & & \\
\hline
\end{tabular}

Tab. 1 Process times for fusion algorithms with different CPU cores

\section{DISCUSSIONS AND CONCLUSIONS}

In the light of the generalized model, this paper presents a parallel framework implementing various fusion algorithms, which not only discards the processing steps and $\mathrm{I} / \mathrm{O}$ operations not impacting the fusion results but also utilizes the parallel computing to improve execution efficiency. The framework can be applied to most fusion algorithms, which usually are divided into three categories, i.e., CS technique, modulation based technique and MRA based technique. Once the framework were established, the main tasks to implement a fusion algorithm are to transform the fusion algorithm to the 
generalized mode and to calculate the last results instead of processing step by step. The experiments show that the parallel processing mechanism in the framework can extremely improve processing efficiency, which can completely utilize the computing resource of the processing platforms ranging from single machine with multi CPU cores to HPC computers. The developed software module can run on different system like Windows, Linux and Mac OS X. Also the experiments show that fusion results using the algorithms in the software are consistent with those of other software.

\section{ACKNOWLEDGEMENTS}

This work was supported by National Natural Science Foundation of China under Grant No. 40901229 and 863 Program under Grant No. 2011AA120401.

\section{REFERENCES}

J. G. Liu, 2000. Smoothing Filter-based Intensity Modulation: a spectral preserve image fusion technique for improving spatial details. International Journal of Remote Sensing, vol.21, pp.3461 3472 .

J. Hill, C. Diemer, O. Stöver, T. Udelhoven, 1999. A Local Correlation Approach for the Fusion of Remote Sensing Data with Different Spatial Resolutions in Forestry Applications. International Archives of Photogrammetry and Remote Sensing, Valladolid, Spain, vol. 32, Part 7-4-3 W6.

J. N’ũnez, X. Otazu, O. Fors, A. Prades, V. c Pal`a, and R.Arbiol, 1999. Multiresolution-Based Image Fusion with Additive Wavelet Decomposition. IEEE Transactions on Geoscience and Remote Sensing, vol.37, pp.1204 1211.

J. Vrabel, 2000. Multispectral imagery advanced band sharpening study. Photogramm. Eng. Remote Sens., vol.66, pp. $73 \sim 79$.

J. W. Carper, T. M. Lillesand, and R. W. Kiefer, 1990. The use of intensity-hue-saturation transformations for merging SPOT panchromatic and multispectral image data. Photogramm. Eng. Remote Sens., vol.56, pp. 459-467.

J. H. Yang, J. X. Zhang, and H. T. Li, 2009. Generalized Model for Remotely Sensed Data Pixel-Level Fusion and Its implementation technology. Journal of Image and Graphics (Chinese), vol. 14, no. 4, pp. $604-614$.

J. H. Yang, J. X. Zhang, et al., 2010. Pixel Level Fusion Methods for Remote Sensing Images: a Current Review, Technical Commission VII Symposium, Vienna, Austria.
J. X. Zhang, J. H. Yang, H. T. Li, et al, 2008. Generalized Model for Remotely Sensed Data Pixel-Level Fusion. The International Archives of the Photogrammetry, Remote Sensing and Spatial Information Sciences, Beijing, China, Vol. XXXVII, Part B7: pp. 1051-1056.

J. X. Zhang, J. H. Yang, Z. Zhao, H. T. Li, and Y. H. Zhang, 2010. Block-regression based Fusion of Optical and SAR Imagery for Feature Enhancement. International Journal of Remote Sensing, Vol. 31, No. 9, pp. 2325-2345.

M. González-Audícana, J. L. Saleta, R. G.. Catalán, et al, 2004. Fusion of Multispectral and Panchromatic Images Using Improved IHS and PCA Mergers Based on Wavelet Decomposition. IEEE Transactions on Geoscience and Remote Sensing, vol. 42, no. 6, pp. 1291 1299.

V. K. Shettigara, 1992. A generalized component substitution technique for spatial enhacement of multispectral images using a higher resolution data set. Photogramm. Eng. Remote Sens., vol. 58, pp. 561-567. 